\title{
LAPAROSCOPIC NEPHRECTOMY IN LIVE DONOR
}

\author{
ANUAR I. MITRE, FRANCISCO T. DÉNES, AFFONSO C. PIOVESAN, FABIANO A. SIMÕES, \\ LÍSIAS N. CASTILHO, SAMI ARAP \\ General Hospital, School of Medicine, University of São Paulo, São Paulo, SP, Brazil
}

\begin{abstract}
Objective: To present the initial experience of videolaparoscopic nephrectomy in live renal donor.

Materials and Methods: In the period from April 2000 to August 2003, 50 left nephrectomies in live donor were performed by videolaparoscopy for transplantation. Twenty-eight patients were male (56\%) and 22 female (44\%). Mean age was 37.2 years, and the mean body mass index (BMI) was $27.1 \mathrm{~kg} / \mathrm{m}^{2}$.

Results: Mean surgical time was 179.5 minutes, and warm ischemia time of the graft was 3.79 minutes. The mean estimated bleeding was $141 \mathrm{~mL}$. There was no need of blood transfusion or conversion to open surgery. In 42 cases (84\%), the vascular portion of the graft was considered good by the recipient's surgical team and in all cases, the ureter was considered of proper size, though in one of them (2\%) its vascularization was considered improper. The transplanted kidneys produced urine still in the surgical room in 46 of the 50 transplantations considered. In only 2 cases opioid was required for analgesia. In average, 3.1 doses of dipyrone were used for each patient during hospital stay, and hospital discharge occurred, in average, after 3.2 days post-operatively. Two patients required re-operations and one of them evolved to death.

Conclusions: The laparoscopic nephrectomy in live donor for renal transplantation is an alternative to conventional open surgery. In relation to the graft, no alteration, either anatomic or functional, was detected. Though there is already a large documentation in the international literature regarding this procedure, in our setting a prospective randomized study with the usual surgical study is still necessary in order to prove the advantages and disadvantages of the method.
\end{abstract}

Key words: kidney transplantation; nephrectomy; living donors; laparoscopy; postoperative complications

Int Braz J Urol. 2004; 30: 22-8

\section{INTRODUCTION}

During kidney transplantation, the organ is usually removed through oblique lumbotomy, a very painful incision that requires a prolonged convalescence period and has esthetical consequences (1).

After 1991, several centers worldwide demonstrated that videolaparoscopic access is superior to the conventional one for performing nephrectomies, in relation to postoperative pain, hospital stay and return to daily activities (2-6). Since 1995, many authors have proposed using the videolaparoscopic method to perform nephrectomy in live donor (7-9). The objective of this study is to present an initial experience with this surgical procedure.

\section{MATERIALS AND METHODS}

Patients were selected as donors by the institution's nephrology team, always respecting the 
following fundamental principles: voluntary wish of the patient concerning the donation, the way it will be performed, and his/her immunological compatibility with the recipient.

Pre-operative assessment of donors included an imaging study of renal vascularization and excretory pathway. For this purpose, ultrasonography of urinary tract, excretory urography, arteriography or magnetic angioresonance were performed. With similar kidneys and equal potential for donation, the left kidney was preferred due to the larger length of renal vein on this side.

It was established, for the purposes of this study, that every patient indicated for donation of left kidney would undergo a videolaparoscopic procedure. When the right kidney was chosen, the patient would undergo a conventional surgical access, through oblique lumbotomy.

In the period from April 2000 to August 2003, 50 nephrectomies in live donor for transplantation were performed by videolaparoscopic approach, all on the left side. In this casuistry, 28 patients were male $(56 \%)$ and 22 female (44\%). The donors' mean age was 37.2 years, ranging from 25 to 60 years, and the mean body mass index (BMI) was $27.1 \mathrm{~kg} / \mathrm{m}^{2}$, with a minimum value of 20.3 and maximum of 35.8 $\mathrm{kg} / \mathrm{m}^{2}$. In 36 cases, there was a kinship relation between donor and recipient. In the remaining 14 cases, even though there was not a direct relationship, preoperative study confirmed immunological compatibility, and the transplantation was performed following judicial authorization.

In 5 cases (10\%), vascular abnormalities were detected: 2 in the pre-operative period and 3 during surgery. In those with pre-operative diagnosis, it was found a double renal artery bilaterally, and an option for the left side was made due to reasons previously exposed. As an intraoperative finding, a case with inferior polar artery was observed, which had not been detected by the arteriography performed before the surgical procedure, one patient with vein duplicity, with ligation of the vessel of smaller diameter being preferred, and in another case, a complex venous malformation was found, with the left renal vein draining to the ipsilateral spermatic vein, and this one draining in turn to the inferior vena cava. In only one case, there was urinary tract malformation, represented by complete ureteral duplicity, corrected by bench surgery following latero-lateral anastomosis of ureters. As antecedents of abdominal surgery, 6 female donors had cesarean births, one patient had undergone a suture of duodenal ulcer 15 years earlier and a female donor had undergone a hysterectomy 13 years earlier.

The donor was admitted on the surgery's eve and the diet was light with no residues. Bowel preparation was not performed. We did not routinely use anticoagulant either. The antibiotic prophylaxis consisted in 1 dose of a first generation cephalosporin at the moment of anesthetic induction that was maintained for 24 hours.

\section{Surgical Technique}

The anesthetic team determined the type of anesthesia. In 43 patients, the procedures were performed under a combination of general intravenous anesthesia and continuous peridural block. In the remaining 7 cases, due to orthopedic limitations in the spine, general intravenous anesthesia was used.

Once the patient was anesthetized, orogastric and urethral catheters were inserted, and maintained throughout the surgery. The patient was positioned in oblique decubitus at $45^{\circ}$ over pads, elevating the left side (Figure-1). The left forearm was fixed in an arch-shape to the surgical table, aiming not to strain the shoulder joint.

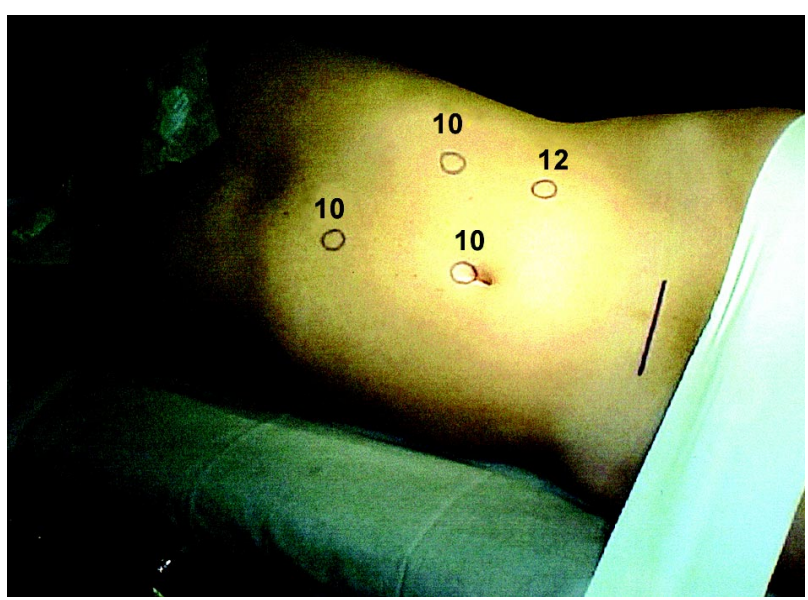

Figure 1 - Patient positioned on the surgical table, with drawings of sites and diameters of trocars (the numbers indicate the diameter in millimeters) and the Pfannestiel incision. 
Donors received in average $2,930 \mathrm{~mL}$ of crystalloid as transoperative hydration. Colloid fluid was not used. Since the beginning of the surgical procedure, manitol $10 \%$ was slowly infused by intravenous line. Before clamping and sectioning the renal pedicle, the manitol infusion was accelerated. Such measures aimed the patient's hyper-hydration, intending to promote an intraoperative diuresis of 2 to $3 \mathrm{~mL} / \mathrm{kg} /$ hour.

The pneumoperitoneum was achieved with a Veress needle, introduced in the upper margin of the umbilical scar. The pneumoperitoneum was maintained in $16 \mathrm{mmHg}$. The first puncture was performed blindly with a disposable 10-mm retractile-tip trocar (Endopath, Ethicon Endosurgery Inc.), in the umbilicus. This trocar served as access for the laparoscope, usually at $30^{\circ}$.

Another puncture in the median line slightly below the xiphoid process, measuring $10 \mathrm{~mm}$, was performed for introduction of the forceps for the assistant. Two other trocars were placed in the left anterior axillary line, with 10 and $12 \mathrm{~mm}$ respectively, for introducing forceps and shears and other instruments for the surgeon. A fifth $15-\mathrm{mm}$ trocar was introduced in the median line, in the middle point of the Pfannestiel transverse suprapubic incision through which the graft was removed (Figure-1). Through this trocar, the plastic bag where the graft was placed after its total dissection (Endocatch II, AutoSuture, USSC) was introduced. The transverse incision did not open the parietal peritoneum, so that there was no escape of the insufflated carbon gas, except during the steps described next.

Following inspection of the peritoneal cavity, the splenic angle and descending colon were widely detached, maintaining the Gerota's perirenal fascia intact. Throughout the procedure, monopolar electrocautery or harmonic scalpel were used for hemostasia.

The upper pole of left kidney was initially and preferentially dissected, separating it from the adrenal gland. The ureter and gonadal vessels were identified, close to the crossover of the iliac vessels. The dissection continued, cranial and always medial to the gonadal vein (aiming to preserve the ureteral vascularization), until the left renal vein. The ligation was then performed with metallic clips, with sub- sequent sectioning of the gonadal vein close to the renal vein, as well as an eventual lumbar vein. Similarly, dissection, clipping and subsequent sectioning of left adrenal vein were performed, largely exposing the supero-anterior aspect of the renal vein on this side. The renal artery was then dissected, close to the aorta, completing the circumferential dissection of the left renal vein. At this moment, the Gerota's fascia was antero-laterally opened, exposing the left kidney surface, which was totally released from the perirenal fat.

The ureter was sectioned close to the crossover with the iliac vessels, as well as the gonadal vein, and the kidney was introduced in the plastic bag. Three $10-\mathrm{mm}$ metallic clips were placed in the renal artery, and the 12-mm mechanical vascular clipper (ETSFlex $35 \mathrm{~mm}$, Ethicon Endosurgery Inc.) was subsequently introduced. In the last 10 nephrectomies, a polypropylene clip (Hem-o-lock, Weck) was used for clamping both renal artery and vein.

Next, and finally, the renal artery was sectioned. The bag was closed and the peritoneal opening of the suprapubic incision was digitally enlarged in order to remove the plastic bag containing the kidney. The trocars were removed and the peritoneal and muscular layers from the 10- and 12-mm orifices were closed with non-absorbable suture passed though a hook needle. Pfannestiel incision was closed in a conventional way. In 3 donors from this casuistry, the incision for removing the kidney was longitudinal suprapubic, over a scar from cesarean birth.

\section{RESULTS}

Mean anesthesia time was 223.1 minutes, with a mean surgical time of 179.5 minutes (ranging from 120 and 270 minutes). Mean warm ischemia time of the graft, considered as the time interval elapsed from the ligation of renal artery until the placement of the graft in an ice-filled container, was 227.4 seconds, oscillating between 72 and $1620 \mathrm{sec}-$ onds (the highest ischemia time was caused by inadvertent ligation of renal artery while ligating the lumbar vein).

Mean estimated bleeding was $141.1 \mathrm{~mL}$ (50 - $350 \mathrm{~mL}$ ). There was no significant bleeding in any 
patient and, consequently, blood transfusions were not required. There was no conversion to open surgery as well. Grafts with an intact renal capsule were obtained in 45 cases, and capsular damages measuring approximately $1 \mathrm{~cm}$ were verified in 5 patients. There was no parenchymal damage requiring suture. In 42 cases $(84 \%)$, the graft vessels were subjectively regarded as long by the recipient's surgical team. In all cases, the ureter was considered of proper size, and in one of them (2\%), its vascularization was considered improper.

Renal perfusion was adequate in all kidneys excepting one, attributed to a renal artery of small diameter. The transplanted kidneys produced urine during the surgery in 46 of the 50 transplantations (92\%).

Donors routinely received sodium dipyrone orally as postoperative analgesic medication. In only 2 cases opioid was required for analgesia, and one of them underwent an exploratory laparotomy on the first post-operative day. In average, 3.1 doses of dipyrone were used for each patient during hospital stay. Patients used an average of 2.7 doses of dipyrone after hospital discharge, which occurred, in average, after 3.2 days postoperatively, ranging from 2 to 6 days.

Two 2 re-operations (4\%) were required. The first one, the 13th case of our experience, it was a male patient, 60 years old, with BMI of $28.7 \mathrm{~kg} / \mathrm{m}^{2}$, donor for his son. Surgery went on without intercurrences, being regarded as difficult due to intestinal gaseous distension. On the second postoperative day, the patient evolved with abdominal distension and nauseas, without fever. At that moment, the laboratory control showed leukopenia with deviation until promyelocyte, and the plain radiography of abdomen confirmed intestinal gaseous distension, with no evidences of pneumoperitoneum. Since the patient's clinical state got worse, an exploratory laparotomy was performed, finding a large amount of fecaloid liquid in the peritoneal cavity and a small perforation in the descending colon. A loop colostomy was performed in the descending colon. The patient evolved with shock refractory to the usual clinical measures and was surgically re-explored on the following day. It was observed a small amount of liquid in the cavity and diffusely poor intestinal perfusion, progressing to death on the seventh postoperative day. The 18th patient, who was male, 36 years old, evolved with abdominal pain, pain in left shoulder and nauseas, without fever or interruption of the intestinal transit. He underwent an exploratory laparotomy that did not reveal any abnormality and present, subsequently, a normal postoperative outcome.

In the recipients, 2 urinary fistulas were seen on the immediate post-operative period due to ureteral necrosis, one in the sixth, and the other in the 17 th postoperative day. Both underwent an anastomosis of the transplanted kidney's pelvis with the recipient's ureter, presenting a good immediate outcome. One of them, however, developed humoral rejection and underwent graft nephrectomy on the 15th postoperative day.

Another patient (2\%) underwent nephrectomy for graft removal with suspected venous vascular thrombosis, which was not confirmed on the pathological examination that demonstrated chronic rejection. Adding both patients, the graft nephrectomy rate was $4 \%$.

The immunosuppressive regimen used in graft recipients consisted in induction with corticoid and azathioprine, introducing cyclosporine as soon as there was a fall in the serum creatinine levels.

In relation to the outcome of the grafts obtained, the laboratory follow-up was performed through dosing of the recipients' serum creatinine. The collection was performed on the day immediately before surgery and, after the procedure, on the first, third, fifth and tenth days. A late dosing was performed as well, 30 days after the transplantation. On the day immediately before surgery, mean creatinine was $8.3 \mathrm{ng} / \mathrm{mL}$ (reference value: 0.5 to $1.7 \mathrm{ng} /$ $\mathrm{mL}$ ), evolving to 4.0 on $1 \mathrm{st}$ PO, 3.4 on 3rd PO; 3.4 on 5th PO and 2.6 on 10th PO. Finally, the late dosing, after 30 days, showed mean creatinine of $1.57 \mathrm{ng} / \mathrm{mL}$ (Figure-2).

The precise assessment of recipients will be the target of a future publication, trying to determine if there is a significant change in outcome when compared with recipients of grafts obtained by open surgery. 


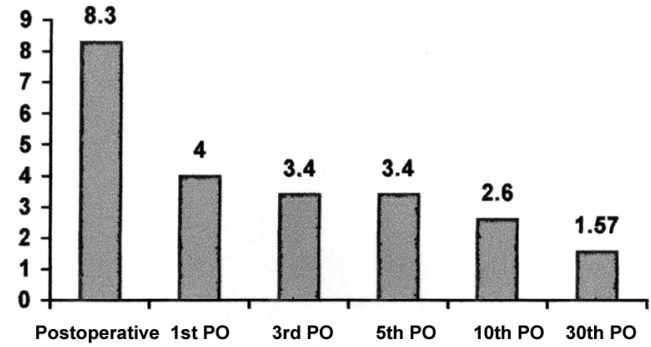

Figure 2-Evolution of the recipients' mean serum creatinine, in relation to postoperative days (in $\mathrm{ng} / \mathrm{mL}$ ).

\section{DISCUSSION}

There is agreement in the literature that the best treatment provided to a patient with terminal chronic renal failure (TCRF) is the renal transplantation, because it increases survival and, fundamentally, the quality of life of these patients (10).

According to data from the Brazilian Society of Organs Transplantation (ABTO), there were in Brazil 21,718 patients in waiting list for renal transplantation by June 2001. This figure represents an increase of $11.7 \%$ in relation to data from December 2000. On the other hand, the same entity predicts that the total number of transplantations performed will remain stable during the current year, what will obviously increase the number of patients under dialysis and, thus, the waiting time for transplantation (10).

Traditionally, the majority of transplantations are performed with organs obtained from cadaveric donors. However, several studies have showed superiority in survival data, both for graft and patient, when kidneys from live donors are used $(5,6,8,9)$. In addition to these factors, the procedure performed from live donors has other advantages over cadaveric donors: the waiting time for transplantation is shorter, which is translated in a shorter period under chronic dialysis. Additionally, the surgical act is performed in an elective way, which represents a better clinical condition for the recipient, increasing the success possibilities of the procedure and shortening the pe- riod of postoperative hospital stay. Finally, it is worthy to remember that, in this kind of donor, a more detailed study for HLA typing is conducted, decreasing the possibility of immunological problems in the posttransplantation period and therefore less immunosuppression is employed. Moreover, a shorter cold ischemia time is achieved, which represents improved survival and early function of the graft $(6,8,9,11)$.

In our setting, there was an increase in the proportion of live donors on the total of transplantations performed, from $48.5 \%$ in 1995 to $57.9 \%$ in 2000 (10). One can observe, however, that such increase is not higher due to potential morbidity factors that are intrinsic to the nephrectomy procedure in live donor. Such factors include prolonged hospital stay and convalescence, causing loss of working days and consequent decrease in the patient's financial gain, postoperative pain and esthetic concerns, naturally associated with the extensive lumbar incision that is normally associated with the donation procedure (5). It must be stressed out that the renal donation in live donor remains as one of the few major surgical procedures that are performed without any physical benefit for the patient. For this reason, since $1995 \mathrm{sev}-$ eral authors (1-7) have proposed the employment of laparoscopic technique for nephrectomy in live donor.

In our setting, we have performed the nephrectomy in live donor by videolaparoscopic approach since April 2000. During this period, we had the opportunity to perform the procedure and to follow the immediate postoperative outcome of 50 patients, all of them being healthy individuals who exerted an altruistic gesture such as donation.

The same anesthetic-surgical team performed the procedure, with some experience accumulated with the use of the videolaparoscopic method. In the first cases conducted, we observed that the removal required more time than that necessary in open surgery. However, we could note that this difference gradually decreased and, in the last procedures, surgical time has been approximately 130 minutes.

There was a death in this casuistry, an uncommon fact in the history of transplantations among live individuals. Analyzing the postoperative outcome and the surgical findings from the urgent exploration 
performed on this patient, we were able to conclude that there was a punctiform intestinal perforation during nephrectomy. The mean rate of intestinal damage during laparoscopic procedures in the literature is 0.5 to $1 \%$ (12). In our patient, such damage failed to be recognized during surgery, which led the patient to fecal peritonitis and the condition of systemic inflammatory response that ultimately caused his death. There were no signs of thermal damage to the bowel, which led us to the hypothesis of a lesion produced by the tip of the Mixter forceps, unprotected by gauze, during withdrawing of the descending colon, since at no time there was direct handling of bowel following its release from the parieto-colic gutter. This poor outcome was determinant for the option of performing a surgical exploration in another patient who presented a clinical picture of abdominal distension associated with pain on the first postoperative day. We believe that there was an overrating of his complaints, since the surgical finding of the exploration was no other than a small amount of serous fluid in left renal cavity. We would probably have observed the patient for a longer period, had not the traumatizing experience of death occurred previously.

Except for these 2 patients, the outcomes were good, with minimal requirements for postoperative and outpatient analgesic medication and early return to routine activities. All patients were satisfied in relation to their expectations about postoperative outcome, concerning both pain and final esthetic result of the surgery. In all cases, the patients would repeat the donation, knowing the postoperative outcome.

The outcome of grafts was also an issue of concern, even though it was not the scope of this study. Some works in the literature $(8,9,11)$ raised the possibility that there was a delay in the evolution of a graft obtained by videolaparoscopic technique. Such delay would occur due to the action of the pneumoperitoneum, which would increase the pressure over the kidney and decrease its filtration capacity following the transplantation. The laboratory outcome analysis of the recipients' serum creatinine did not confirm this hypothesis. Actually, there was a progressive decrease in creatinine levels following the transplantation, similarly to what is seen after a conventional nephrectomy.

\section{CONCLUSIONS}

Though our experience is still initial, laparoscopic nephrectomy in live donor for renal transplantation is a well-systematized procedure that provided a good postoperative outcome for the vast majority of patients. Its performance, though, requires a significant experience in videolaparoscopic surgery, considering the surgical load and potential risks for the live donor.

\section{REFERENCES}

1. Sasaki TM, Finelli F, Bugarin E, Fowlkes D, Trollinger J, Barhyte DY, et al.: Is laparoscopic donor nephrectomy the new criterion standard? Arch Surg. 2000; 135: 943-7.

2. Fabrizio MD, Ratner LE, Montgomery RA, Kavoussi LR: Laparoscopic live donor nephrectomy. Urol Clin North Am. 1999; 26: 247-56.

3. Kavoussi LR: Laparoscopic donor nephrectomy. Kidney Int. 2000; 57: 2175-86.

4. Leventhal JR, Deeik RK, Joehl RJ, Rege RV, Herman $\mathrm{CH}$, Fryer JP, et al.: Laparoscopic live donor nephrectomy - is it safe? Transplantation. 2000; 70: 602-6.

5. Schweitzer EJ, Wilson J, Jacobs S, Machan CH, Philisophe B, Farney A, et al.: Increased rates of donation with laparoscopic donor nephrectomy. Ann Surg. 2000; 232: 392-400.

6. Ratner LE, Montgomery RA, Maley WR, Cohen C, Burdick J, Chavin KD, et al.: Laparoscopic live donor nephrectomy: the recipient. Transplantation. 2000; 69: 2319- 23.

7. Ratner LE, Ciseck LJ, Moore RG, Cigarroa FG, Kaufman HS, Kavoussi LR: Laparoscopic live donor nephrectomy. Transplantation. 1995; 60: 1047-9.

8. Kuo PC, Johnson LB, Sitzmann JV: Laparoscopic donor nephrectomy with a 23-hour stay: a new standard for transplantation surgery. Ann Surg. 2000; 231: 772-9.

9. Berney T, Malaise J, Mourad M, Morel P, Squifflet JP: Laparocopic and open live donor nephrectomy: a cost/benefit study. Transpl Int. 2000; 13: 35-40.

10. Brazilian Register of Transplants: Official Organ of the Brazilian Society of Organs Transplantation (ABTO). June 2001; Year VII, number 2. [in Portuguese]

11. Litwin DE, Darzi A, Jakimowicz J, Kelly JJ, Arvidsson D, Hansen P, et al.: Hand-assisted laparoscopic sur- 
gery (HALS) with the HandPort system: initial experience with 68 patients. Ann Surg. 2000; 231: 715-23.

12. Vallancien G, Cathelineau X, Baumert H, Doublet JD,
Guillonneau B: Complications of transperitoneal laparoscopic surgery in urology: review of 1,311 procedures at a single center. J Urol. 2002; 168: 23-6.

Received: October 18, 2002

Accepted after revision: January 12, 2004

\section{Correspondence address:}

Dr. Anuar Ibrahim Mitre

Disciplina de Urologia, Hospital das Clínicas

Rua Dr. Enéas de Carvalho Aguiar, $255 / 7^{\circ}$ andar

São Paulo, SP, 05403-000, Brazil

Fax: + $55113064-7013$

E-mail: anuar@mitre.com.br 